\title{
El orden de las cosas: mujeres educadoras y desigualdad de génepo en la educación superior*
}

\author{
Ana Magdalena Solís Calvo \\ Universidad de Ciencias y Artes de Chiapas, Tuxtla Gutiérrez, Chiapas, México \\ anamsoliscalvo@hotmail.com
}

\section{RESUMEN}

En el presente artículo se retoman algunos principios expuestos por organismos internacionales (Banco Mundial, Unesco, entre otros), que marcan ruta en las actuales políticas públicas en el ámbito educativo con relación a los términos de inclusión y desigualdad en América Latina. La finalidad es revisar las implicaciones del discurso del liberalismo y el capitalismo como principios fundantes de la educación para todos, donde las mujeres son incluidas, pero no tienen las mismas condiciones que los hombres.

Se muestra el caso de dos grupos de estudiantes mujeres de la licenciatura en Educación Preescolar en la Unidad 097 Sur de la Universidad Pedagógica Nacional, en Ciudad de México. Estos casos sirven como referente para cuestionar los elementos imbricados en las políticas educativas actuales y la manera en que las mujeres han ocupado un lugar de desigualdad estructural desde la conformación de la organización de las

sociedades, el origen de la democracia y las fusiones patriarcales del capital y los Estados liberales. Al retomar un estudio de caso documentado a través de una investigación cualitativa desde la perspectiva sociocultural y al tener como principal herramienta de indagación las entrevistas a profundidad, las narrativas autobiográficas de las estudiantes dan cuenta de la condición de género y las implicaciones socioculturales, familiares, económicas en las que viven estas mujeres que son a la vez docentes y estudiantes. Los datos empíricos presentados son tamizados por referentes teóricos, entre ellos los feministas, para develar la desigualdad estructural de género vedada bajo las promesas de inclusión educativa en las actuales políticas públicas en el ámbito educativo.

Palabras clave: política educativa; educación superior; formación docente; desigualdad; género; teorías feministas.

Cómo citar: Solís Calvo, A. M. (2020). El orden de las cosas: mujeres educadoras y desigualdad de género en la educación superior. Ciencias Sociales y Educación, 9(17), 83-93. https://doi.org/10.22395/csye.v9n17a4 Recibido: 15 de diciembre de 2019.

Aprobado: 12 de marzo de 2020. 


\section{The Order of Things: Educator Women and Gender Inequality in Higher Education}

\section{ABSTRACT}

In this article, we retake some principles exposed by international organizations (World Bank, Unesco, among others), that made routes for contemporary public policy in the realm of education with relation to the terms of inclusion and inequality in Latin America. The goal of this article is to review the implications of the liberal and capitalist discourses as guiding principles of education for all and where women are not included but have the same conditions as men.

We display the case of two groups of women students from the undergraduate program in pre-school education in the 097 South Unit of Universidad Pedagógica Nacional (National Pedagogic University) in Mexico City. These cases serve as referents for questioning the element involved in the current educational policies and how women have occupied a place of structural inequality since the conformation of the organization of societies, the origin of democracy, and the patriarchal fusions and intricacies between capital and liberal States.

By retaking a case study documented through qualitative research from the socialcultural perspective and by having in-depth interviews as the main tool of inquiring, the autobiographical narratives of the female students give an account of the gender conditioning and their social and cultural, family-related, and economic repercussions lived by these women that are teachers and students at the same time. The empirical data presented here are sieved by theoretical references, the feminist ones among them, for unveiling the structural gender inequality underlying in the promises of educational inclusion in the current public policy for the educational realm.

Keywords: educational policy; higher education; teachers education; inequality; gender; feminist theories.

\section{A ordem das coisas: mulheres educadoras e desigualdade de gênero no ensino superior}

\section{RESUMO}

Neste artigo, são retomados alguns princípios expostos por organismos internacionais (Banco Mundial, Unesco, entre outros) que determinam o caminho das atuais políticas públicas no contexto educativo quanto à inclusão e à desigualdade na América Latina. O objetivo é verificar as implicações do discurso do liberalismo e do capitalismo como princípios fundantes da educação para todos, em que as mulheres são incluídas, mas não têm as mesmas condições do que os homens. Apresenta-se o caso de dois grupos de estudantes mulheres da licenciatura em Educação Pré-escolar na Unidade 097 Sul da Universidad Pedagógica Nacional, na Cidade do México. Esses casos servem como referente para questionar os elementos envolvidos nas políticas educativas atuais e a maneira em que as mulheres ocupam um lugar de desigualdade estrutural a partir da conformação da organização das sociedades, da origem da democracia e das fusões patriarcais do capital e dos Estados liberais. Ao retomar um estudo de caso documentado por meio de uma pesquisa qualitativa, sob a perspectiva sociocultural, e ao ter como principal ferramenta de questionamento as entrevistas a profundidade, as narrativas autobiográficas das estudantes evidenciam a condição de gênero e as implicações socioculturais, familiares, econômicas nas quais essas mulheres, docentes e estudantes ao mesmo tempo, vivem. Os dados empíricos apresentados são depurados por referentes teóricos, entre eles os feministas, para revelar a desigualdade estrutural de gênero vedada sob as promessas de inclusão educativa nas atuais políticas públicas no âmbito educativo.

Palavras-chave: política educativa; ensino superior; formação docente; desigualdade; gênero; teorias feministas. 


\section{Introducción}

El presente documento se desprende de una investigación amplia realizada por un equipo de investigadores interesados en la formación de mujeres educadoras (Solís, Sánchez y Lira, 2016). Después de analizar varios elementos de la condición de género en la educación superior, de manera particular nace en mí la inquietud de volver sobre los datos recabados en aquella investigación mayor y retomar algunos de ellos. En principio, esto responde a una necesidad personal, pues como mujer me identifico con ciertas condiciones de género. La segunda motivación está ligada a mi formación profesional como docente en esta licenciatura, por ende, me interesa conocer a las estudiantes participantes en este estudio. Con estos antecedentes, la finalidad de tomar un nuevo sesgo investigativo es mirar nuevamente los datos obtenidos a través de diversas teorías feministas para la construcción de elementos de análisis en la interpretación de las desigualdades de género que constituyen el espacio académico de la universidad. En este caso, se analiza una institución pública de Ciudad de México a cargo de la formación de licenciadas en educación preescolar. Las condiciones de esta institución pueden ser similares a otras instituciones de educación superior (IES) en Latinoamérica.

El primer apartado se ocupa de exponer uno de los problemas que abordaré en este documento: las actuales políticas educativas en materia de inclusión. En el segundo apartado se presentan las condiciones particulares de dos grupos de mujeres educadoras que cursan la licenciatura en Educación Preescolar en la Universidad Pedagógica Nacional en Ciudad de México, que fue la institución donde se llevó a cabo este estudio. Esta institución se encarga de profesionalizar por medio de la señalada licenciatura a docentes de educación preescolar que imparten clases en este nivel educativo sin tener un título de educación superior que avale su profesión.

Por último, la discusión se centra en revisar lo que constituye la esfera privada y los principios fundantes del liberalismo que sostienen las actuales tendencias en educación, para volver a poner sobre la mesa los discursos de las últimas tres décadas de las políticas públicas en esta materia. Con ello, pretendo generar una discusión sobre cómo la construcción del orden de las cosas - se hace una analogía con la condición natural que ocupa la mujer en la organización desigual—, ha sido una tradición heredada desde la Grecia antigua. Espero que los elementos discutidos en este escrito den cuenta de los elementos estructurales implicados en la construcción social de la desigualdad de género que se ocultan en los discursos de las políticas educativas inclusivas. En este contexto, las condiciones económicas, familiares, socioculturales, de formación y desigualdad educativa de las estudiantes, quedan inadvertidas. 


\section{Las políticas educativas: inclusión y desigualdad}

La Escuela, tal como la conocemos hoy en día, es el producto de procesos sociales que se sostienen sobre principios liberales: "los individuos como seres libres e iguales, emancipados de los vínculos asignados y jerarquizados de la sociedad tradicional" (Pateman, 1996, p. 2). En la búsqueda de que este principio de igualdad en materia educativa se establezca como derecho universal, algunos organismos internacionales como la Organización de las Naciones Unidas para la Ciencia, la Educación y la Cultura (Unesco) y la Organización de Estados Iberoamericanos (OEI), entre otras, han declarado "educación para todos" (Unesco, 1990, 2000) para garantizar que los gobiernos de los países que los integran proporcionen educación de calidad a sus habitantes, sin excepción alguna.

Los países pertenecientes a estas organizaciones han concretado acciones diversas en el marco de la cooperación internacional. En la franja de Iberoamérica el pronunciamiento más reciente y ambicioso en este sentido son las Metas Educativas 2021. En este documento se establecen diez metas para garantizar una educación de calidad a todos los ciudadanos de estos países para alcanzar su principal propósito que consiste en:

[...] lograr que más alumnos estudien, durante más tiempo, con una oferta de calidad reconocida, equitativa e inclusiva [...] asegurar el derecho a la educación de calidad desde la primera infancia, la cobertura universal y gratuita de la primaria y secundaria y a mejorar las condiciones de vida y las oportunidades reales de las y los jóvenes, que permitan su crecimiento integral para lograr mayores niveles de inclusión y desarrollo social en nuestros países. (OEI, 2010, p. 25)

Desde estas políticas de desarrollo se considera que el papel de la Escuela y la educación básica es indispensable para soslayar las cuestiones de desigualdad existentes en los países de la región pertenecientes a la Organización de Estados Iberoamericanos (OEI). Sin embargo, las cuestiones de desigualdad que se viven en estas naciones tienen que ver con la diversidad de condiciones sociales y económicas que enfrentan sus habitantes, donde las situaciones de desigualdad y pobreza no pueden ser en ningún sentido igualitarias.

Los esfuerzos realizados en políticas públicas dirigidas a la educación son coherentes con el papel central que ella cumple como eslabón del desarrollo. La educación y el empleo son reconocidos como ámbitos privilegiados de superación de los problemas sociales, operando como mecanismos de inclusión social, de reducción de desigualdades y de superación de la pobreza. (OEI, 2010, p. 35)

Estas políticas públicas aseguran que, al mejorar la calidad y la equidad en la educación, se hará frente a la pobreza y a la desigualdad para favorecer la inclusión social. La inclusión desde esta visión significa garantizar educación 
para todos; lo que ha llevado a las políticas en materia educativa a generar estrategias que aseguren la extensión de la cobertura en educación básica. En consecuencia, en los últimos siete sexenios en México, se han emprendido reformas educativas. Con la llegada de una nueva administración, se implementa otra reforma a la educación nacional sin comprender las necesidades que tienen todos a los que se desea incluir; las condiciones de vida particulares; si todos hablan español como primera lengua; si en la comunidad las niñas y los niños tienen los mismos derechos o se regulan por cuestiones de género que los distinguen en oportunidades; si existen características físicas que les impiden integrarse a la escuela; si las situaciones económicas, familiares y sociales que se presentan en la comunidad o el contexto favorecen o complican la integración al sistema educativo nacional.

La inclusión y la exclusión, desde la mirada de Popkewitz (1991), no son conceptos opuestos, sino mutuamente imbricados y los discursos más inclusivos califican y descalifican a la gente para participar. En este mismo sentido, en el año 2015, la Comisión Económica para América Latina y el Caribe (Cepal) reportó que ciento setenta y cinco millones de personas en Latinoamérica viven en pobreza. Esta situación impide que la gente más vulnerada por su condición económica tenga acceso y permanencia en los sistemas educativos nacionales, no solo en educación básica, sino en niveles de educación media y superior. Esta cifra revela que la inclusión educativa en los países de la región es una meta que aún está lejos de ser lograda.

Una de las instituciones de Educación Superior (IES) en México que atiende a la población más vulnerable en nuestro país es la Universidad Pedagógica Nacional. Además de ofrecer diversas licenciaturas, atiende la formación inicial y continua de los docentes del país. Los programas de esta institución son del interés particular en el escenario anteriormente descrito, debido a que esta universidad profesionaliza al personal que se desempeña como docente en educación preescolar pero que solo tiene estudios a nivel media superior.

\section{Condiciones de las mujeres educadoras que estudian en la Unidad 097 Sur de la Universidad Pedagógica Nacional de Ciudad de México}

En la Universidad Pedagógica Nacional, la licenciatura en Educación Preescolar Plan 2008 (LEP 08) tiene como propósito profesionalizar a las personas que laboran en este nivel y que no cuentan con la formación superior para desempeñarse como docentes. Es importante señalar que este programa se diseñó para maestros en servicio. Por tanto, no se aceptan estudiantes egresados de bachillerato si no presentan constancia de que ejercen la profesión docente frente a un grupo (Solís, Sánchez y Lira, 2016). 
Este programa de licenciatura considera en su diseño curricular la incorporación el uso de las tecnologías de la información y la comunicación (TIC) debido a que cada cuatrimestre se cursan dos asignaturas en línea. La implementación del uso de la tecnología en las IES es otra medida de las recientes reformas educativas. Con estas se pretende la inclusión de las estudiantes en lo que se denomina la sociedad del conocimiento para dar respuesta a las necesidades de formación del siglo XXI (OEI, 2010).

Las características del diseño instrumental de dicha licenciatura, descritas en el párrafo anterior, no consideran las condiciones de la población que atiende. La mayoría de las estudiantes ha tenido una historia de vida escolar y laboral fragmentada: vuelven a la escuela después de no estudiar por varios años y tienen que utilizar la tecnología sin recursos económicos ni habilidades para ello. Este hecho muestra las condiciones de vulnerabilidad de estas estudiantes, quienes están en riesgo de abandonar la formación profesional.

La metodología para realizar la indagación parte de una perspectiva sociocultural para conocer las necesidades de atención que tienen nuestras alumnas y elaborar las primeras clasificaciones que permitan hacer una caracterización de algunas de sus trayectorias formativas, condiciones sociales, económicas, familiares, pero no solo desde elementos sociodemográficos. La intención fue recabar esta información a través de sus propias narrativas, vertidas en entrevistas y autobiografías, que constituyeron las principales herramientas de investigación de corte etnográfico. En la tabla 1 se muestran de manera general las condiciones encontradas en treinta y cinco alumnas de dos grupos en la LEP 08 (Solís, Sánchez y Lira, 2016).

Los datos expuestos en la tabla 1 fueron recogidos en las entrevistas a profundidad y las narrativas autobiográficas llevadas a cabo durante la recopilación de información de campo. La información muestra que la mayoría de las estudiantes de la LEP 08 tienen más de veintiún años al ingresar y los rangos de edad son amplios: el grupo mayoritario está conformado por personas entre los 31 y 41 años, en contraste con los últimos grupos de edad que están conformados por seis profesoras entre 42 y 51 años y tan solo tres alumnas con más de 52 años. 
Tabla 1. Características de la población

\begin{tabular}{|c|c|c|c|c|c|}
\hline \multirow[t]{3}{*}{ Características de la población } & \multirow{2}{*}{\multicolumn{5}{|c|}{ Edades }} \\
\hline & & & & & \\
\hline & 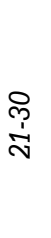 & 워 & 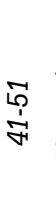 & \multicolumn{2}{|c|}{ 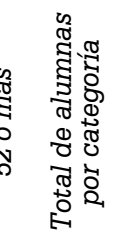 } \\
\hline Soltera & 2 & 1 & 0 & 0 & 3 \\
\hline Viven en pareja & 4 & 6 & 4 & 2 & 16 \\
\hline Jefas de familia & 6 & 7 & 2 & 1 & 17 \\
\hline Recién egresadas de bachillerato & 3 & 0 & 0 & 0 & 3 \\
\hline Con más de cinco años sin estudiar & 7 & 10 & 0 & 0 & 17 \\
\hline Con más de diez años sin estudiar & 2 & 4 & 6 & 3 & 15 \\
\hline Se considera usuaria hábil y sin problemas de acceso y conectividad & 4 & 2 & 0 & 0 & 6 \\
\hline $\begin{array}{l}\text { Se considera usuaria medianamente hábil y tiene algunos problemas de } \\
\text { acceso y conectividad }\end{array}$ & 6 & 4 & 2 & 0 & 12 \\
\hline Se considera usuaria poco hábil y no tiene acceso ni conectividad & 2 & 8 & 4 & 3 & 17 \\
\hline Trabaja con sueldo y prestaciones de ley & 0 & 10 & 2 & 2 & 14 \\
\hline Trabaja con condiciones menores de sueldo y sin prestaciones & 12 & 4 & 4 & 1 & 21 \\
\hline
\end{tabular}

Fuente: Solís, Sánchez y Lira (2016).

La diversidad de edades es una de las características que distingue a la población de primer ingreso en la licenciatura en Educación Preescolar de la Universidad Pedagógica Nacional en México, la cual está diseñada exprofeso para profesionalizar a docentes en servicio. Esto es solo un indicador que muestra la diversidad de trayectorias de formación educativa y de experiencias laborares de las estudiantes de acuerdo con su edad. En un mismo grupo de primer semestre hay estudiantes que ejercen como docentes y que son recién egresadas de bachillerato, en contraste con las estudiantes/docentes del último rango de edad (52 años y más), quienes superan los veinte años de experiencia laboral.

Las entrevistas y las narrativas autobiográficas recogidas durante la indagación revelaron que los procesos de escolarización son fragmentarios en algunos casos, si tomamos el rango de tiempo que han dejado de estudiar, es decir, entre cinco y diez años. A ello agregamos que las fracturas en la escolarización han sido múltiples, desde situaciones de reprobación de materias hasta identificar que la secundaria o el nivel de bachillerato lo han realizado por medio de exámenes únicos que les acreditan el nivel (Ceneval). Esto es un 
dato significativo porque para muchas de estas mujeres la existencia de estas formas de acreditar conocimiento les ha permitido continuar estudiando, lo cual, en principio, les da acceso a la educación superior con base en su experiencia laboral. Sin embargo, en ciertas cuestiones académicas las pone en desventaja con relación a otras compañeras con estudios escolarizados, por ejemplo, en el uso de herramientas tecnológicas, competencias de lectura y redacción; aunque la formación escolarizada tampoco es garantía de ello.

Con relación al uso de las tecnologías, hemos señalado que la licenciatura incorpora dos asignaturas donde se requiere el manejo de las TIC. Con respecto a esto, la tabla 1 revela que tan solo seis estudiantes/docentes se perciben hábiles para su manejo. Esta situación coloca, indudablemente, a las veintinueve estudiantes restantes en condiciones desiguales para integrarse al trabajo en la universidad. Otras cuestiones que acentúan estas desigualdades de inclusión son que de las treinta y cinco alumnas, veintiuna perciben salarios bajos y nulas prestaciones. Además, solo tres son solteras en contraste con diecisiete de ellas que son jefas de familia (Solís, Sánchez y Lira, 2016).

En cuanto a las desigualdades sobre el uso de la tecnología, es importante señalar el fenómeno de la primera brecha digital (Pedró, 2009) que se considera de acuerdo con la ubicación geográfica de los usuarios, el nivel de acceso y conectividad. En este sentido, es necesario discurrir que la capacidad para utilizar, aprovechar y apropiarse de las TIC en los países latinoamericanos, entre ellos México, presenta situaciones desfavorables (OCDE, 2016).

Para Castaño (2008), también existe una segunda brecha digital que se traduce en exclusión social de género, al marcar diferencias y desigualdades en los usos y habilidades que tienen hombres y mujeres con respecto a la tecnología. Estas diferencias afectan especialmente a las mujeres y supone "una barrera que impide su plena incorporación a la sociedad de la información" (Castaño, 2008 , p. 10). Estas diferencias quedan expuestas en la tabla 1, donde se puede observar que al ser estudiante mujer existen condiciones distintas de género: el cuidado de los hijos; ser el sostén de la familia, ya sea de los descendientes o de los padres; cumplir con obligaciones de aseo doméstico u otros condicionamientos de género que representan limitaciones tanto para estudiar como para laborar. Además, los sueldos de las mujeres en América Latina son veintitrés por ciento más bajos que los de los hombres según la Organización Internacional del Trabajo (OIT, 2016).

Esta situación se opone a las políticas públicas que promueven la educación para todos, a sus principios educativos modernos, los cuales establecen una equivalencia entre igualdad y homogeneidad que congela las diferencias (Dussel, 2000). 
La visión de que todos somos iguales en cuanto al derecho genera una ceguera que niega las condiciones diferentes e individuales de cada ser humano. Lo importante de ofrecer oportunidades de educación para todos no es el concepto de inclusión, sino las prácticas de equidad educativa. En especial, es importante la inclusión en las IES donde las condiciones de las mujeres son de desigualdad en estructuras de jerarquización social. No desde la modernidad, sino más atrás, esta exclusión basada en el género deviene de una tradición heredada.

\section{El orden de las cosas. La desigualdad de género: una tradición heredada}

Los estudios de género han revelado que el origen de la desigualdad hacia las mujeres está en la dicotomía de lo público y lo privado. Pateman (1996) recupera la visión clásica de los griegos donde la familia constituía una forma de organización desigual en la que el padre tenía todo el poder sobre su mujer, sus hijos y sus esclavos, por lo que estos quedaban sometidos a su control al considerarse parte de su propiedad privada. En este sentido, Arendt (1958) afirma que "dentro de la esfera doméstica la libertad no existía" (p. 234). En este modelo de organización social, las relaciones entre iguales se establecían en la polis del orden de la esfera pública a la que pertenecían solo los hombres.

El poder del patriarcado anula a las mujeres como individuos dada su condición de propiedad (Duby, 1985). A finales del siglo XX, al vincularse el régimen capitalista con los ideales liberales, la condición de la mujer, que devenía de la esfera privada bajo el nuevo régimen económico, se consideró como una propiedad más del patriarca. De esta forma, se le negaron los derechos que el espacio público brinda a los hombres desde el liberalismo. Se le otorgó la condición de ser dependiente e incapaz de tomar decisiones debido a su naturaleza femenina que tiene relación con la vida privada/familiar (Rabotnikof, 1998, p. 11). Fue excluida de las cuestiones públicas al considerarla carente de razón y cultura, constituyentes exclusivos de naturaleza masculina.

Las condiciones sociales y de género de las mujeres educadoras que realizan sus estudios en la UPN son determinadas por las esferas de lo público y lo privado que, como señala Rabotnikof (1998), han transformado sus límites históricamente de acuerdo con las formas en que la vida se ha concebido desde lo social y lo político. En este escenario, la educación para todos, entendida como acceso y cobertura, no va a dar respuesta ante las necesidades de estas mujeres estudiantes en Latinoamérica, debido a la desigualdad educativa. Para la población más desfavorecida, son pocas las oportunidades de acceso a la educación superior que se tienen en la región.

Según la OEI (2013), el panorama de la formación de los docentes latinoamericanos es precaria (doce años de educación). La educación en Latinoamérica 
es débil, en comparación con la de Estados Unidos y Japón que es de dieciséis años. Además, en los países de América Latina se atienden a los alumnos con las peores condiciones socioeconómicas de este bloque económico que asisten a escuelas donde los maestros son improvisados. En este mismo sentido, Vaillant (2004, p. 54) expone que en los países pobres, como es el caso de México, el reclutamiento de los docentes se realiza cada vez más desde sectores con menor nivel educativo y económico. Esta situación se ha mostrado, por ejemplo, en el caso de estas estudiantes/docentes que provienen de sectores precarizados de Ciudad de México.

\section{Conclusiones}

Las reflexiones vertidas en este espacio permiten discutir que no es posible considerar a todos los estudiantes en igualdad de condiciones y que es necesario establecer nuevas políticas en las IES públicas que no solo les permitan ingresar, sino permanecer y terminar sus estudios de educación superior. Esto no podrá llevarse a cabo mientras se siga interpretando la inclusión como sinónimo de igualdad. Esta idea cubre la condición de desigualdad de género de las mujeres que deviene de la conformación de la organización social en un Estado que situó a las mujeres en el ámbito de lo privado y que, hasta nuestros días, las continúa colocando en relación de desigualdad en el ámbito educativo.

Considero que un punto nodal de investigaciones subsecuentes será conocer de manera profunda cuáles son las condiciones que tienen de manera particular las mujeres educadoras que se profesionalizan en las aulas de la UPN. Será necesario revelar los retos que enfrentan al ingresar a la universidad de acuerdo con las diversas esferas sociales en las que intervienen desde su lugar como hijas, madres, estudiantes, educadoras, sus edades, responsabilidades familiares y profesionales, entre otras. Esto es indispensable para contribuir al diseño de políticas educativas que favorezcan la inclusión educativa a partir de las diferencias. Con ello, se propende por una inclusión que no quede a nivel discursivo, sino que ponga en marcha programas educativos que consideren la desigualdad como una cuestión estructural que está conformada en una complejidad social que implica problemas del orden económico. La pobreza y la condición de género para las mujeres que estudian en situaciones cada vez más vulnerables, perpetúan el orden de las cosas. Así, se dan situaciones limítrofes que difícilmente se pueden subsanar con una mera intención de proporcionar oportunidades educativas, por más incluyentes que estas pretendan ser. 


\section{Referencias}

Arendt, H. (1958). La condición humana. Paidós.

Castaño, C. (2008). La segunda brecha digital. Ediciones Cátedra.

Cepal. (2015). Panorama social de América Latina. https://www.cepal.org/sites/default/files/ presentation/files/220321_ps_2015_ppt.pdf

Duby, G. (1985). Obertura. Poder privado, poder público. En P. Aries y G. Duby (eds.), Historia de la vida privada (pp. 21-54). Aries.

Dussel, I. (2010). La reproducción de la exclusión en el aula: una revisión de la escuela moderna en América Latina. Flacso.

OCDE. (2016). Perspectiva de la OCDE en Ciencia, Tecnología en innovación en América Latina (Extractos). https://www.oecd-ilibrary.org/science-and-technology/perspectivas-de-la-ocde-en-ciencia-tecnologia-e-innovacion-2016-extractos_9789264303546-es

OIT. (2016). Las mujeres en el trabajo. Tendencias 2016. Organización Internacional del trabajo Unesco.

OEI. (2010). Metas educativas 2021. La educación que queremos para la generación de los bicentenarios. Organización de Estados Iberoamericanos.

OEI. (2013). Miradas sobre la educación en Iberoamérica. Desarrollo profesional docente y mejora de la educación. Organización de Estados Iberoamericanos para la Educación, la Ciencia y la Cultura.

Pateman, C. (1996). Críticas feministas a la dicotomía público/privado. En C. Castells (comp.). Perspectivas feministas en teoría política. (pp. 2-23). Paidós.

Pedró, F. (2009). Are the new millennium learners making their grades? Technology use and educational performance in PISA. OECD-Centre for Educational Research and Innovation (CERI).

Popkewitz, T. (1991). A Political Sociology of Educational Reform. Teacher's College Press.

Rabotnikof, N. (1998). Público privado. Debate Feminista, 18 (pp. 3-13). http://www.debatefeminista. cieg.unam.mx/wp-content/uploads/2016/03/articulos/018_01.pdf

Vaillant, D. (2004). Construcción de la profesión docente en América Latina. Tendencias, temas y debates.

Solís, A., Sánchez, J. y Lira, J. (2016). Crear una comunidad de aprendizaje con mujeres educadoras en formación. Revista Didac, 68, 19-26. http://revistas.ibero.mx/didac/articulo_detalle.php?id_ volumen $=22 \&$ id_articulo $=276 \&$ id_seccion $=128 \&$ active $=127 \&$ pagina $=18$

Unesco. (1990). Declaración Mundial sobre Educación para Todos. https://unesdoc.unesco.org/ark:/48223/ pf0000184556

Unesco. (2000). Foro Mundial sobre la Educación para Todos. https://unesdoc.unesco.org/ark:/48223/ pf0000121147_spa 